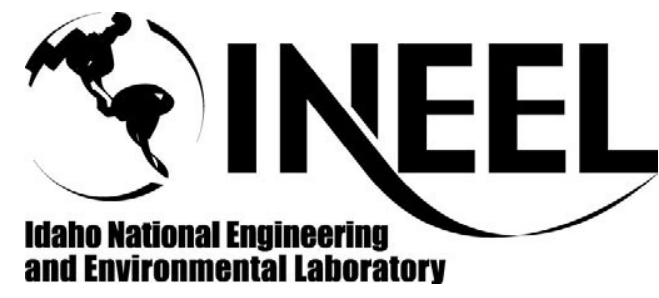

INEEL/CON-04-01559

PREPRINT

Material Property Measurement In Hostile Environments Using Laser Acoustics

\author{
Ken L. Telschow
}

August 24, 2004

2004 IEEE International Ultrasonics, Ferroelectrics and Frequency Control

This is a preprint of a paper intended for publication in a journal or proceedings. Since changes may be made before publication, this preprint should not be cited or reproduced without permission of the author.

This document was prepared as an account of work sponsored by an agency of the United States Government. Neither the United States Government nor any agency thereof, or any of their employees, makes any warranty, expressed or implied, or assumes any legal liability or responsibility for any third party's use, or the results of such use, of any information, apparatus, product or process disclosed in this report, or represents that its use by such third party would not infringe privately owned rights. The views expressed in this paper are not necessarily those of the U.S. Government or the sponsoring agency. 


\title{
Material Property Measurement in Hostile Environments using Laser Acoustics
}

\author{
Ken L. Telschow* \\ Idaho National Engineering and Environmental Laboratory \\ Idaho Falls, ID 83415-2209, USA
}

Abstract - Acoustic methods are well known and have been used to measure various intrinsic material properties, such as, elastic coefficients, density, crystal axis orientation, microstructural texture, and residual stress. Extrinsic properties, such as, dimensions, motion variables or temperature are also readily determined from acoustic methods.

Laser acoustics, employing optical generation and detection of elastic waves, has a unique advantage over other acoustic methods - it is noncontacting, uses the sample surface itself for transduction, requires no couplant or invasive sample surface preparation and can be utilized in any hostile environment allowing optical access to the sample surface. In addition, optical generation and detection probe beams can be focused to the micron scale and/or shaped to alter the transduction process with a degree of control not possible using contact transduction methods. Laser methods are amenable to both continuous wave and pulse-echo measurements and have been used from $\mathrm{Hz}$ to 100 's of $\mathrm{GHz}$ (time scales from sec to psec) and with amplitudes sufficient to fracture materials.

This paper reviews recent applications of laser acoustic methods for determining material properties in hostile environments that preclude the use of contacting transduction techniques. Example environments include high temperature $(>1000 \mathrm{C})$ sintering and molten metal processing, thin film deposition by plasma techniques, materials moving at high velocity during the fabrication process and nuclear high radiation regions. Recent technological advances in solid-state lasers and telecommunications have greatly aided the development and implementation of laser acoustic methods, particularly at ultra high frequencies. Consequently, laser acoustic material property measurements exhibit high precision and reproducibility today. Optical acoustic imaging is both quantitative and rapid.

\section{INTRODUCTION}

Acoustic wave propagation in materials has been shown to be a very effective probe of material extrinsic (dimensions, temperature,...) and intrinsic (elastic stiffness, density, residual stress, phase transformations,...) properties. Materials processing and nondestructive evaluation (NDE) are two industrial fields that widely use acoustic methods for noninvasive inspection. Most acoustic NDE today is focused on detecting critical flaws, such as cracks, in materials to help predict when failure might occur. However, a much better approach would be to monitor material properties and, in particular, microstructure properties in a fundamental way that can predict the state of a material along its "road to failure", as depicted in figure 1.

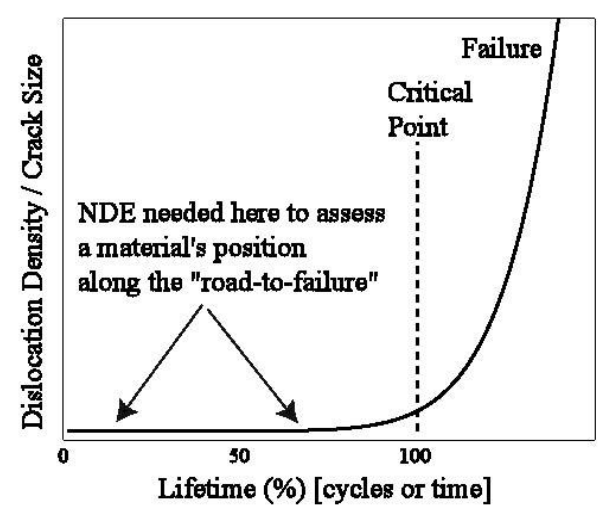

Figure 1. Typical "road to failure" of a material.

In order to monitor material properties during the processing stage and / or during use, a noncontacting and noninvasive methodology must be used. This often precludes the use of contact transducers, such as piezoelectric, that alter the properties measured 
(loading) or are not usable in the often hostile environments (high temperature). Laser acoustics offers a viable method that can be used in almost any hostile environment that allows optical access to the material surface. Lasers are used to both generate and detect elastic wave motion at the surface of materials and the technique is now a well established method for performing elastic wave measurements that does not require physical contact with the sample. [1] Typically, a pulsed laser delivers a high intensity but very short impulse to the sample through absorption of light at the surface. Operation in the thermoelastic regime does no damage to the material and is well understood $[2,3]$, but usually much less efficient than operation in the ablation regime. [4]

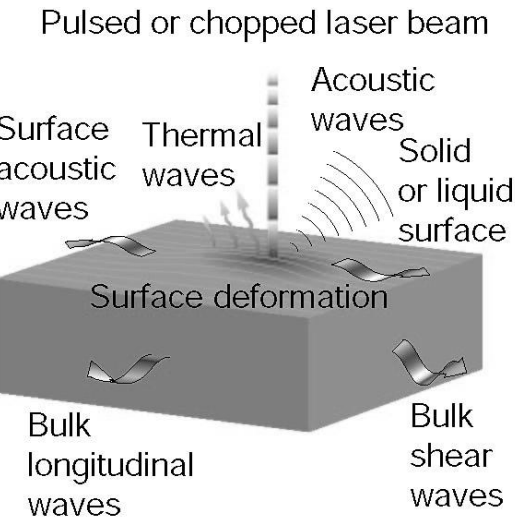

Figure 2. Generation of elastic wave modes in a material from absorption of light at the surface.

An optical interferometer serves for detection by demodulating the small phase modulation that the elastic wave motion produces on a continuous probe laser beam. [5,6] Dynamic interferometric detection is particularly useful as it provides absolute calibration of acoustic displacement (referenced to the wavelength of light), exhibits extremely wide bandwidths (limited only by the detection electronics), can be employed even from materials with high optical absorption (graphite) or with rough industrially prepared surfaces. The latter ability comes from using self-referencing interferometers, such as the passive Fabry-Perot or the active Photorefractive. Both of these detection techniques automatically account for multiple speckles reaching the photodetector, thereby providing high light gathering capability for measurements on high attenuating materials.

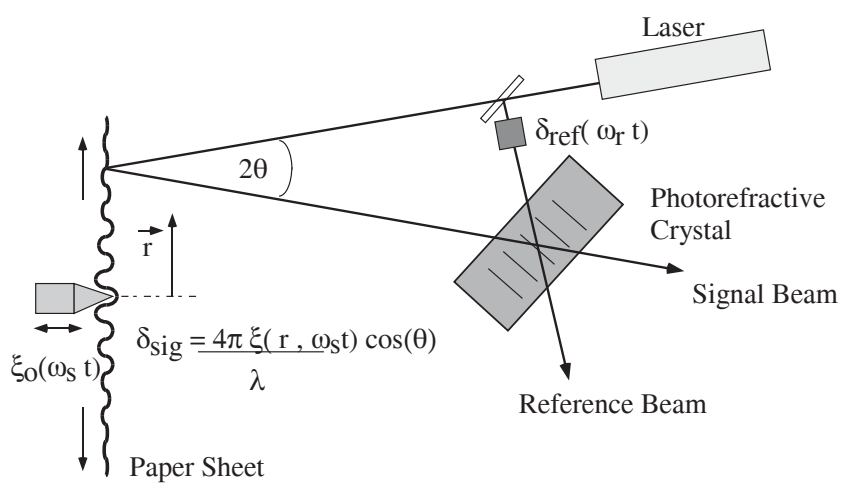

Figure 3: Interferometric technique for acoustic motion detection utilizing the Photorefractive effect.

Laser sources can be readily altered to enhance the generation process by controlling the spot diameter and location on the sample surface. Similarly, the detection process can be easily scanned on the surface to provide spatial information about the wave propagation process. Since, wide bandwidth is inherent in the whole process, laser sources can more readily be used at frequencies much higher than normal contact piezoelectric sources. Laser acoustics has been used at $\mathrm{GHz}$ frequencies to image modes in thin film resonators through scanning. [7,8,9]

Interferometry using the photorefractive effect can be easily expanded to not only adapt automatically to environmental noise but also to perform full-field imaging without scanning. [10] Imaging is particular appealing as information is obtained in both the spatial and wavenumber domains simultaneously, which can greatly help interpretation of the results.

Macroscopic measurements involve those where the acoustic wavelength is much larger than any microstructural feature (e.g. grain, pore, inclusion, etc.,). These measurements necessarily average over the microstructure of the material.

Microscopic measurements involve scattering and transmission through individual features that are comparable to the acoustic wavelength. This regime of operation provides microstructure information on a fundamental level.

Laser acoustic methods operate in both regimes and provide control over all variables that determine spatial and temporal resolution and are applicable to all materials through the ability to control the optical wavelength employed. 


\section{MACROSCOPIC APPLICATIONS}

\section{Ceramic Sintering}

Ceramic sintering involves heating ceramic powders, consolidated to a very porous "green" to temperatures greater than $1000 \mathrm{C}$ for extended periods of time. Throughout this heating process, the powder particles fuse and eventually consolidate into a solid material with little or no porosity (i.e. full density). Laser acoustics was used to study this process in-situ on a $\mathrm{ZnO}$ ceramic pellet. [11] Excitation was accomplished by directing a laser pulse onto one side of the pellet, which detection was through Fabry-Perot interferometry with a different laser beam directed on the opposite pellet face.

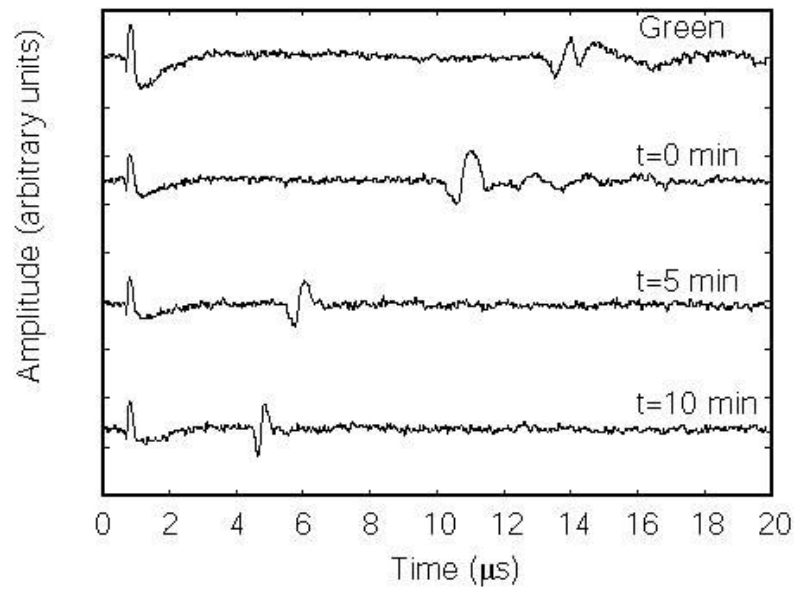

Figure 4. Real-time laser acoustic measurements in through transmission of the sintering process for $\mathrm{ZnO}$ at $850 \mathrm{C}$.

Through transmission waveforms taken during the sintering process at $850 \mathrm{C}$ are shown in figure 4 . The sintering process at this temperature took about 1 hour to complete. Initially, the waveform showed a very diffuse acoustic signal that changed into a well defined narrow acoustic pulse as the part sintered. Eventually, the waveforms became similar to those seen in fully consolidated parts and multiple echoes were observed. Throughout this process a significant change in acoustic velocity was observed that essentially tracked the consolidation process as shown in figure 5 .

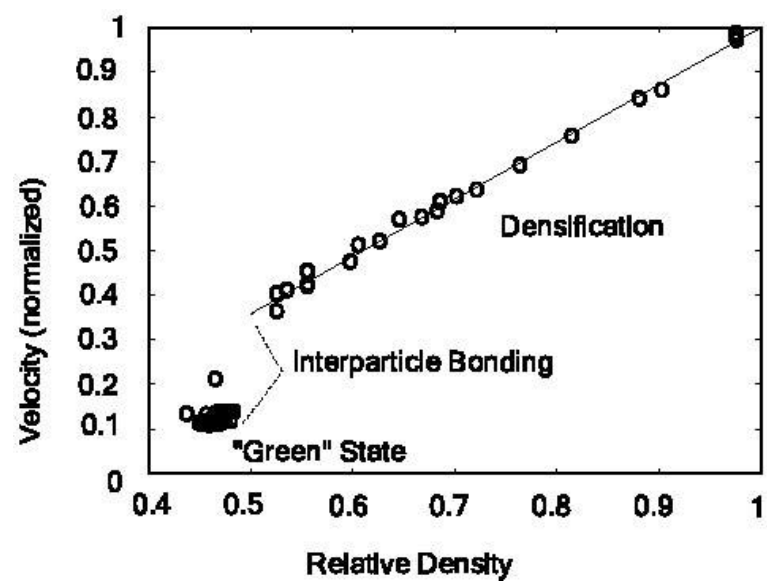

Figure 5. Through transmission longitudinal wave velocity vs. consolidation of $\mathrm{ZnO}$ pellets sintered to temperatures of $800 \mathrm{C}$ to $960 \mathrm{C}$.

\section{Molten Metal Processing}

Figure 6 shows acoustic echoes obtained from the solidification interface in a molten metal. [12] Titanium is routinely purified by heating in a crucible to temperatures greater than $1600 \mathrm{C}$ for times long enough for inclusions to dissolve.

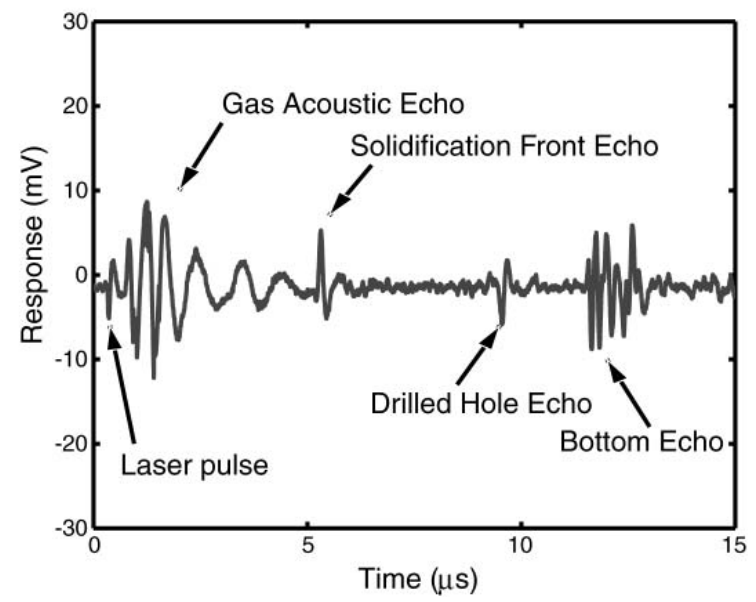

Figure 6. Acoustic echoes of a wave generated at the liquid surface and reflected from the solidification interface, a calibration hole and the solidified metal.

The laser acoustic method allowed measurements to be made directly below the plasma torch, as depicted in figure 7 . The high temperatures involved and the demands for the highest purity of titanium prohibit the use of other acoustic sensing methods in this case. 


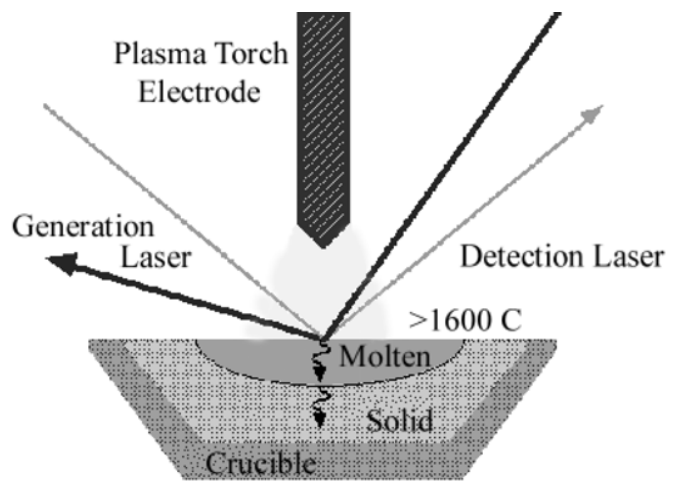

Figure 7. Schematic depicting laser generation and detection in a pool of molten titanium.

\section{High Nuclear Radiation Environment}

A particularly hostile environment is the interior of a nuclear reactor. The need exists for monitoring degradation of materials in-situ to the nuclear reactor and even the core so that critical states of materials and parts are known at all times. Laser acoustics provides a unique method that would be applicable to this environment if light can be directed to and from the interior.

A proof-of-principle test was recently performed in the Advanced Test Reactor at the INEEL. The fuel storage canal has an experiment access tube (gamma tube) that allows placement of a sample into the high gamma radiation flux. A laser based acoustic system was constructed that both generated and detected acoustic motion in a part located within the gamma tube from a remote point near the edge of the canal.

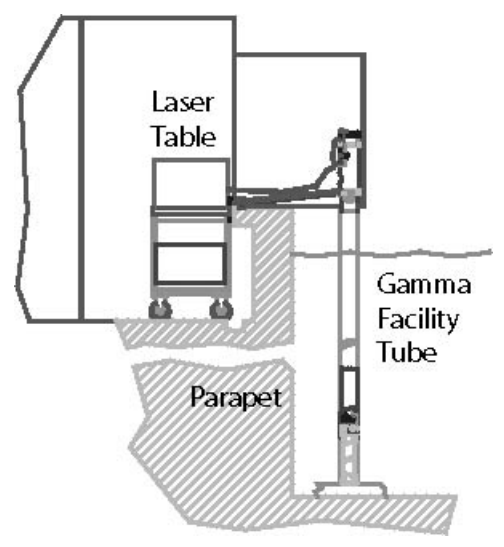

Figure 8. Setup for the laser acoustic demonstration in the gamma tube at the INEEL.
A sample holder and mirror positioning system was constructed to insert the sample into the gamma tube and place it in the high flux region. Bulk reflecting optics was utilized to maneuver both the generation laser beam $(532 \mathrm{~nm})$ and the detection laser beam $(1064 \mathrm{~nm})$ onto the sample surface, while providing a means to collect the light reflected from the sample for detection. A photorefractive interferometer automatically suppressed room vibration effects and readily detected subnanometer acoustic displacements at frequencies up to several MHz. Figure 8 shows a schematic of the arrangement with the laser equipment on a movable cart next to the canal. The sample was located near the bottom of the gamma tube about 25 feet away from the laser table.

Laser based resonant ultrasound spectroscopy was chosen for this initial test as this alleviated stringent requirements on the positioning of the laser beams on the sample. The sample was held at 3 points, 2 top and 1 bottom, allowing independent positioning of both laser beams along the outer cylindrical surface, see figure 9 .

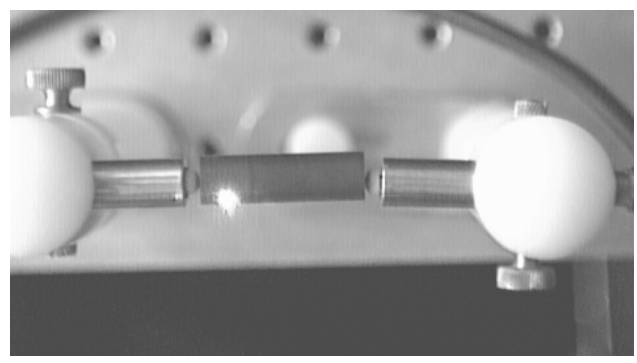

Figure 9. Cylindrical sample showing the detection laser beam at $532 \mathrm{~nm}$.

Many vibration modes of the cylindrical sample steel were recorded with each laser pulse. The data was recorded over a period of about 170 hours several times a day. During this time, the gamma flux was increased in three steps from $10^{4}$ to $10^{6}$ Roentgen $/ \mathrm{hr}$. Figure 10 shows the measured frequency for a split bending mode of the sample along with the sample temperature as measured by an thermocouple in the sample holder.

The effect of the gamma flux was to mainly heat the sample, as shown by the rise in the thermocouple measurement. Simultaneously, the resonant modes of the sample decreased, following the temperature. Analysis of these results indicated that the decrease in the resonant mode frequency with temperature is a 
result of the temperature dependence of the elastic stiffness for this material. Clearly, this simple test showed the efficacy of the laser acoustic technique for measuring change sin material properties in-situ to high radiation fields and in real-time.
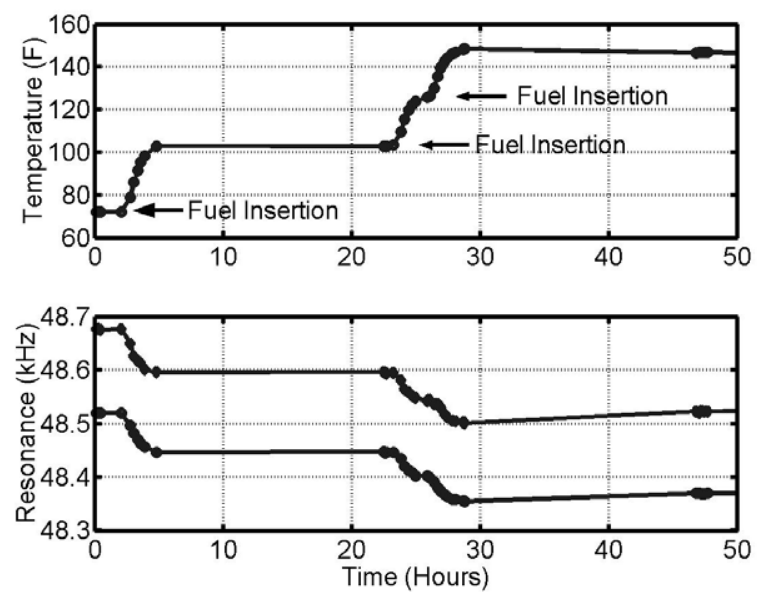

Figure 10. Sample temperature (top) and frequencies for a split bending mode (bottom) as a function time showing the times when additional fuel was placed near the gamma tube to increase the flux.

\section{Full-field imaging of acoustic motion}

Full-field interferometric processing of images is possible using the photorefractive effect. [13] Twowave mixing of signal and reference beams within these materials can produce an output beam that demodulates the phase difference into an intensity variation. The photorefractive effect also automatically produces a quadrature phase shift between signal and reference beams when the charge transport mechanism is diffusive [14]. Another desirable feature of photorefractive interference processing is that complicated optical wavefronts from surfaces with rough features are readily accommodated through the volume holographic process that produces the optimum reference beam for interferometric detection (i.e. samples with rough surfaces can be investigated with little loss of sensitivity due to speckle averaging). Full-field imaging of acoustic motion over the entire surface of a sample have been are recorded at video frame rates, not requiring scanning over the surface, through a variant of the photorefractive two-wave mixing scheme. $[15,16]$
A schematic of the optical detection process is shown in Figure 11. A solid state single frequency

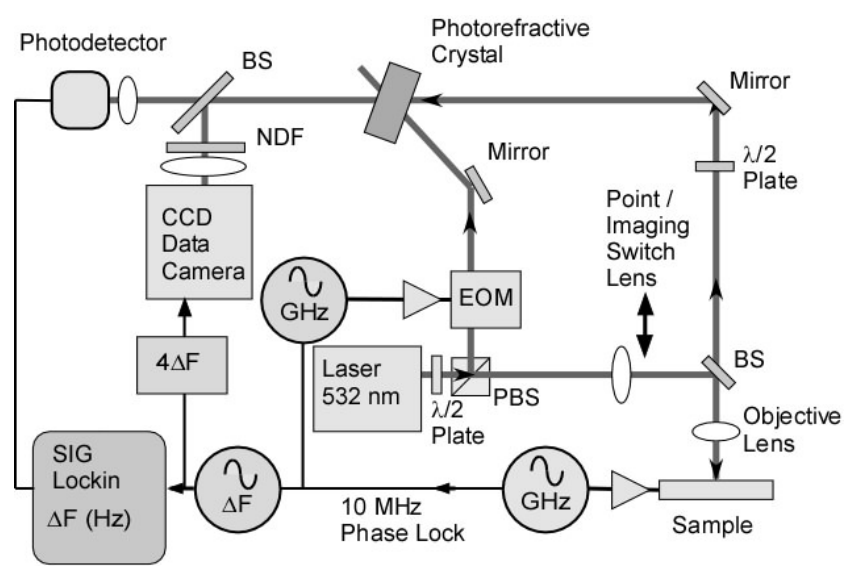

Figure 11. Schematic of the photorefractive interferometer apparatus for full-field imaging and single point detection.

laser at $532 \mathrm{~nm}$ produces both the signal and reference beams. After suitable beam expansion, the sample is illuminated with the signal beam. A picture of the sample surface is imaged inside a photorefractive material along with the reference beam. The two beams are combined in a Bismuth Silicon Oxide (BSO) photorefractive crystal, operated in the charge diffusion mode. The resultant output beam from the two-wave mixing is then measured with a CCD video camera for full-field imaging or with a photodiode detector and lock-in amplifier for single point measurement. The CCD captures the intensity and phase information between the signal and reference images into a single image. The signal beam is modulated by the surface motion of the ultrasonic wave and an external electrooptic modulator is used to modulate the phase of the reference beam such that the difference frequency is at a value suitable for the optical detector (CCD or photodetector); this heterodyne process allows the technique to be used at any frequency. Any single frame has information on the wave propagation in all planar directions. This is most easily seen in the Fourier transform of the image, which produces a plot of the inverse wavelength (wavenumber) in all directions.

Measurements on paper were performed by driving a flexural wave from a point piezoelectric transducer, as before. The microstructure of paper and the elastic 
stiffness matrix are anisotropic as seen in the wavefront pattern of figure 12 and also in the transformed wavenumber image of Figure 13.

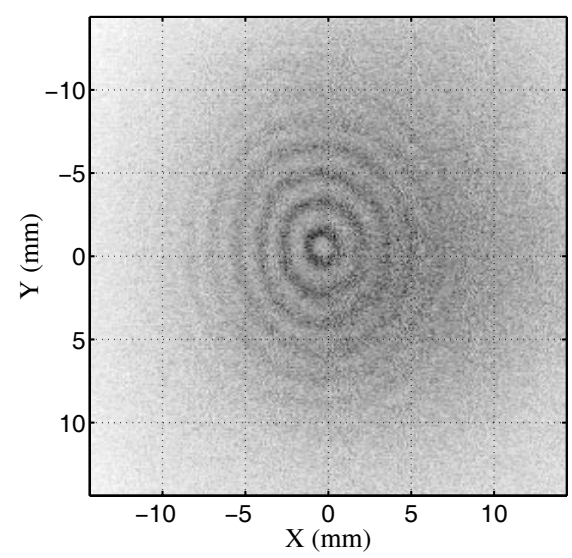

Figure 12. Single frame image of the lowest flexural mode in raw stock paper at $100 \mathrm{kHz}$.

Note that the ultrasonic wavefronts are clearly shown in all directions at once over $20 \times 20 \mathrm{~mm}$ region. Inphase and quadrature images were used to produce a complete map of acoustic amplitude and phase at all points on the paper surface within view. The anisotropic nature of the ultrasonic wave propagation is clearly visible in the images as well as laser speckle.

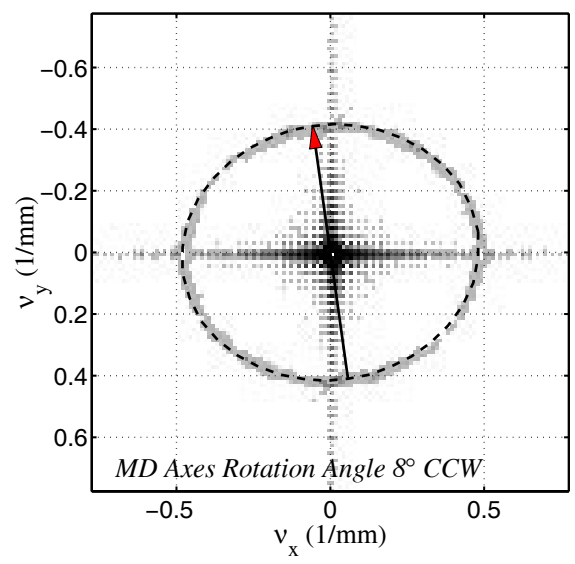

Figure 13. Transform image of the raw stock paper image showing the anisotropic wavenumber for this paper sample. The dashed line is the theoretical calculation assuming the MD axis is vertical.
Fourier transform of the displacement image results in an image of the "wavenumber" diagram for this material; that is, an image showing $(1 / \lambda)$ in all directions, where $\lambda$ is the ultrasonic $\mathrm{A}_{0}$ mode wavelength at the measurement frequency. Processing the spatial image in this manner is particularly convenient as it utilizes all the data the image provides. Even images with much reduced signal to noise ratio still result in useable images for comparison to the theoretical predictions. Superimposed onto the FFT image of Figure 12 is the prediction resulting from an exact theoretical calculation that is valid for all planar directions in an orthotropic sheet. There are no adjustable parameters in this comparison. The theoretical predictions are based entirely on the elastic coefficients, density and thickness parameters obtained from independent measurements. The exceptional agreement between the theoretical predictions and the experimental data from the camera measurements is readily apparent and testifies to the overall accuracy of the entire laser acoustic measurement process. [17]

\section{MICROSCOPIC APPLICATIONS}

The ability with laser acoustics to control the spot size down to the micron range, makes the technique well suited for microscopic environments. Both the single point and full-field imaging measurements have been adapted to a microscope at the INEEL for measurements on the scale of individual features of a material's microstructure. Measurements on the scale of individual grains in a material, allow investigation of the interaction of elastic waves with grain boundaries at a very fundamental level.

\section{Grain boundary interaction with surface waves}

Initial tests have been performed to investigate linear and nonlinear effects of surface wave propagation through an Aluminum grain boundary. [18] An aluminum sample of high purity was annealed to allow large grains of $\mathrm{mm}$ size to grow, which allowed generation and detection within and between individual grains. A tone burst from the generation laser beam was split into two parts that were recombined at the sample surface to produce a grating interference pattern as shown in figure 14 . The modulated intensity distribution of the grating 
generated surface waves with a wavelength equal to the fringe spacing, $\sim 21 \mu \mathrm{m}$ at about $140 \mathrm{MHz}$.

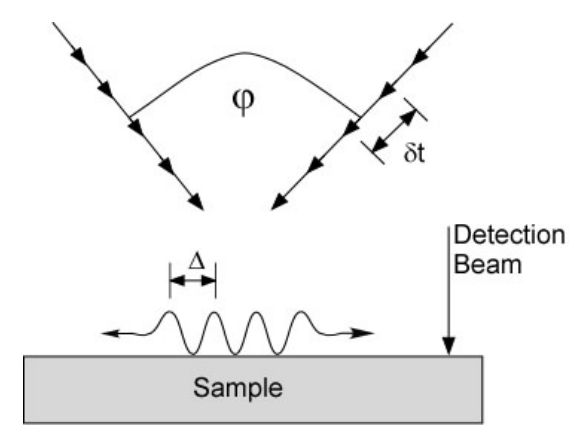

Figure 14. Coupled two-beam interference to produce surface waves of a specific wavelength, $\Delta=21 \mu \mathrm{m}$ and $\delta \mathrm{t}=7 \mathrm{~ns}$.

The surface wave was measured with a Michelson interferometer at various points along a line that passed through three grain boundaries, as shown in the insert to figure 15 . At each point the signal was Fourier analyzed to record the fundamental and $2^{\text {nd }}$ harmonic. The ratio of these signal amplitudes is plotted in the figure as a function of distance along the line. Increases in the harmonic amplitude ratio were observed near all three grain boundaries.

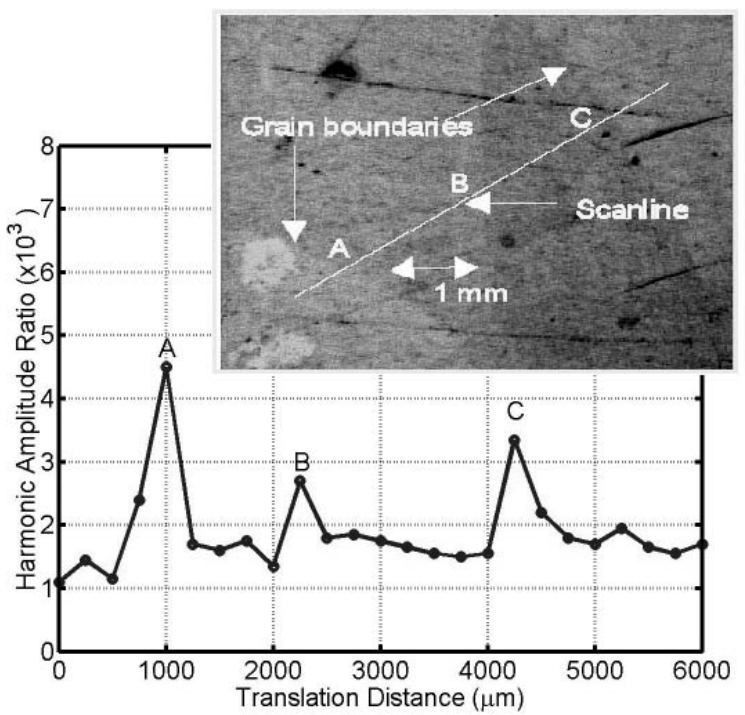

Figure 15. Harmonic amplitude ratio scan involving three grains showing increased ratio near the grain boundaries $(\mathrm{A}, \mathrm{B}, \mathrm{C})$.

Laser acoustics at the microscopic level is an important tool for the study of elastic wave interactions with material microstructure. It can form a quantitative microscopic method for characterizing features, such as grain boundaries and dislocations.

\section{Microscopic full-field imaging of plate waves}

The full-field imaging technique has also been adapted to a microscope geometry. This allows images of acoustic motion with a field of view down to about $50 \mu \mathrm{m} \times 50 \mu \mathrm{m}$, within which the spatial resolution is better than $1 \mu \mathrm{m}$. Figure 16 shows the inphase image of a lateral mode excited in a thin film acoustic resonator at $844 \mathrm{MHz}$. [19] The thin film resonator consists of a free-standing layer of piezoelectric Aluminum Nitride (AlN) with Molybdenum (Mo) coatings on the top and bottom. The primary series resonance is at $875 \mathrm{MHz}$, but around this value lateral plate wave modes are readily excited whose wavenumber can be obtained directly by inspecting the Fourier transform image, shown to the right in the figure. It can be seen that four lateral modes are simultaneously excited.
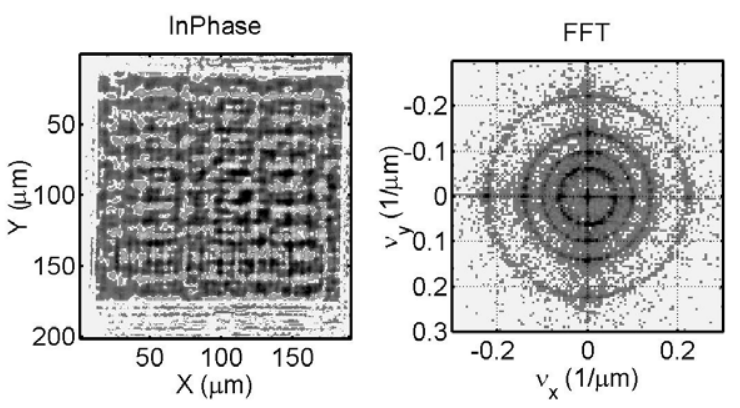

Figure 16. Image of a lateral mode vibration in a thin film acoustic resonator (left) and its Fourier transform in the wavenumber regime (right).

The identity of these modes becomes apparent when the dispersion curve for plate waves in anisotropic Mo/AlN/Mo plates is calculated. A calculation of the wave mode dispersion relation for the multilayered plate was performed using partial wave analysis. [20] Figure 17 shows a calculation of the two lowest symmetric (S0, S1) and antisymmetric (A0, A1) modes in the Mo/AlN/Mo plate, AlN (not including piezoelectricity) in the Wurtzite structure with the c-axis perpendicular to the plate surface and 
the Mo layers oriented with the [111] direction normal to the surface.

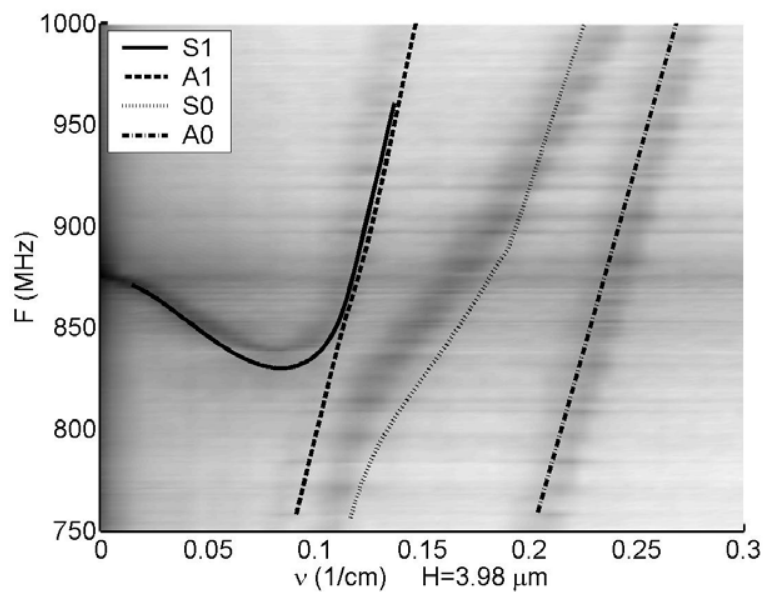

Figure 17. Compilation Fourier transforms of lateral mode vibrations over a large frequency region (image) with theoretical predictions of dispersion for the first four plate wave modes.

The thickness of the AlN layer used in the calculation was chosen so that the series resonance (thickness expansion mode in the normal direction) was at the experimentally determined frequency of $875 \mathrm{MHz}$. This results in a total thickness for the plate of $3.98 \mu \mathrm{m}$, which compares reasonably well with the experimentally determined total thickness of 3.80 $\mu \mathrm{m}$, using the experimentally determined Mo thickness. The main features of the measured dispersion curve are reproduced rather well in the calculation which allows identification of the modes as the lowest four plate wave modes, S1, A1, S0, A0. Reasonably good quantitative agreement was obtained between the measurement and the theoretical prediction using the experimentally determined film thickness and material parameters taken from the literature. These results show the quantitative nature of the laser acoustic imaging method that allows detailed comparison with theoretical prediction.

\section{CONCLUSIONS}

Laser methods offer a large adaptability for making acoustic measurements in solids, liquids and gases. The ability to control the excitation process spatially and temporally allows a great diversity in the generation of elastic waves. All wave modes (bulk longitudinal, bulk shear, surface waves, plate waves, ...) can be readily generated with the thermoelastic optical absorption process. Utilizing the ablation region, high intensity laser pulses can produce impulses strong enough to generate nonlinear surface waves with enough energy to cause materials to fail. $[21,22,23]$

Laser acoustic detection employs optical interferometers, some which have been adapted to work with rough surfaces and in industrial environments. Control over the detection spot size allows use at microscopic as well as macroscopic dimensions. Imaging ultrasound with a laser ultrasonic camera employing photorefractive dynamic holography has been described that is capable of investigating the elastic behavior of isotropic and anisotropic materials in several ways. The camera allows one to observe ultrasonic motion in real-time at video frame rates using heterodyning of the motion to slower speeds.

The laser acoustic method is capable of flat frequency response over a wide range and is fully functional on samples of complex materials and rough surfaces. Successful implementation of laser acoustic measurements has been shown in severely hostile environments where no contact with the sample is possible using conventional transducers. Laser acoustics has also been shown to quantitative, providing the ability to make absolute displacement and wave propagation parameter measurement.

\section{ACKNOWLEDGEMENTS}

The author is indebted to Dave Cottle, Rob Schley, John Walter, Dave Hurley, Vance Deason and Scott Watson from the INEEL for their assistance in performing the many measurements outlined in this paper. The author also thanks Professor Subhendu Datta and O. Mukdadi of the University of Colorado for the modeling calculations, and Dr. J. Gerhartstein, Dr. P. Brodeur and Dr. C. Habeger of the Institute of Paper Science and Technology, Atlanta, GA, for the elastic constant matrices of the paper samples. The thin film acoustic resonators were produced by Agilent Technologies Inc. and the help of Dr. John Larson III is greatly appreciated in obtaining the 
lateral mode images. This work was sponsored by the U.S. Department of Energy, Office of Energy Research, Office of Basic Energy Sciences, Engineering Research and the INEEL Laboratory Directed Research \& Development program under DOE Idaho Operations Office Contract DE-AC0799ID13727.

\section{REFERENCES}

1 C. B. Scruby, R. J. Dewhurst, D. A. Hutchins and S. B. Palmer, "Laser Generation of Ultrasound in Metals," Research Techniques in Nondestructive Testing, vol.Ê2, R. S. Sharp editor (Academic press, N.Y., 1982), 281-327.

2 V. E. Gusev and A. A. Karabutov, Laser Optoacoustics, (A. I. P. New York, 1993)

3 F. V. Bunkin, A. A. Kolomensky and V. G. Mikhalevich, Lasers in Acoustics, (Harwood Publisher Academic GmbH, Chur, Switzerland, 1991)

4 R. J. Conant, K. L. Telschow and J. B. Walter, "Mathematical Modeling of Laser Ablation In Liquids With Application To Laser Ultrasonics," Ultrasonics 40, 1067-1077 (2002).

5 J.-P. Monchalin, "Optical Detection of Ultrasound," IEEE Trans. on UFFC $\underline{33}$, 485-490, (1986).

6 J. W. Wagner, "Optical Detection of Ultrasound," Physical Acoustics, Vol. XIX, Eds. Thurston, R. N., and Pierce, A.D., (Academic Press, NY, 1990) Chp. 5.

7 J. V. Knuutila, P. T. Tikka and M. M. Salomaa, "Scanning Michelson interferometer for imaging surface acoustic wave fields," Opt. Lett. 25, 613 (2000).

8 J. E. Graebner, B. P. Barber, P. L. Gammel, D. S. Greywall and S. Gopani, "Dynamic visualization of subangstrom high-frequency surface vibrations," Appl. Phys. Lett. 78, 159-161 (2001).

9 Ken L. Telschow, Vance A. Deason, David L. Cottle and John D. Larson III, "UHF Acoustic Microscopic Imaging of Resonator Motion" 2000 IEEE Ultrasonics Symposium Proceedings, S.C. Schneider, M. Levy, B. R. McAvoy eds., IEEE, Piscataway, NJ. Vol.1, 631-634 (2000).

10 K.L. Telschow, V. A. Deason, R. S. Schley and S. M. Watson, "Direct Imaging of Lamb Waves in
Plates using Photorefractive Dynamic Holography," J. Acoust. Soc. Am. 106(5), 25782587 (1999).

11 K. L. Telschow, J. B. Walter, G. V. Garcia, "Laser Ultrasonic Monitoring of Ceramic Sintering," J. Appl. Phys. 68 (12), 1990, 6077-6082.

12 J. B. Walter, K. L. Telschow and R. E. Haun, "Laser acoustic molten metal depth sensing in titanium," Advanced Sensors for Metals

Processing, eds. B. W. Brusey, J. F. Bussière, M. Dubois and A. Moreau, (Canadian Institute of Mining, Metallurgy and Petroleum, Montreal, Quebec, 1999) 265-274

$13 \mathrm{P}$. Yeh, Introduction to Photorefractive Nonlinear Optics, (John Wiley, New York, 1993).

14 R. K. Ing and J. P. Monchalin, "Broadband optical detection of ultrasound by two-wave mixing in a photorefractive crystal," Appl. Phys. Lett. 59, 3,233 (1991).

15 K. L. Telschow, V. A. Deason, R. S. Schley and S. M. Watson, "Direct Imaging of Lamb Waves in Plates using Photorefractive Dynamic Holography," J.Acoust. Soc. Am. 106(5), 25782587 (1999).

16 K. L. Telschow, V. A. Deason, D. L. Cottle, and J. D. Larson III, "Full-Field Imaging Of GHz Film Bulk Acoustic Resonator Motion," IEEE_Trans. Ultrason., Ferroelect., Freq. Contr., 50(10), 12791285 (2003)

17 O. Mukdadi, S. K. Datta, K. L. Telschow and V. A. Deason, "Ultrasonic Guided Waves in Thin Orthotropic Layers: Theoretical Analysis and Dynamic Holographic Imaging Measurement," accepted for publication in the journal IEEE Transactions: Ultrasonics, Ferroelectrics and Frequency Control (2001).

18 D. H. Hurley and K. L. Telschow, "Probing Acoustic Nonlinearity on Lengths Scales Comparable to Material Grain Dimensions," Ultrasonics 40, 617-620 (2002).

19 K. L. Telschow, V. A. Deason, D. L. Cottle, and J. D. Larson III, "Full-field Imaging of Acoustic Motion at Nanosecond Time and Micron Length Scales," 2002 IEEE Ultrasonics Symposium Proceedings, October 8-11, Munich, Germany, D. Yuhas and S. Schneider, eds., IEEE, Piscataway, NJ. Vol.1, 601-604 (2002).

20 K.L. Telschow, "Laser Acoustic Imaging Of Film Bulk Acoustic Resonator (FBAR) Lateral Mode 
Dispersion," to be published in Reviews $O f$

Progress In Quantitative NDE, Vol. 23, Edited By

D. O. Thompson And D. E. Chimenti (2005

American Institute of Physics).

21 A. Lomonosov, V. G. Mikhalevich, P. Hess, E.

Yu. Knight,M. F. Hamilton and E. A.

Zabolotskaya, "Laser-generated nonlinear

Rayleigh waves with shocks," J. Acoust. Soc.

Am. 105 (4), 2093-2096 (1999).

22 A. M. Lomonosov and P. Hess, "Impulsive

Fracture of Silicon by Elastic Surface Pulses with

Shocks," Phys. Rev. Lett. 89(9), 095501-1-4 (2002).

23 S. Catheline, J.-L. Gennisson, M. Tanter, and M. Fink, "Observation of Shock Transverse Waves in Elastic Media," Phys. Rev. Lett. 91(16), 164301-14 (2003).

* Ken Telschow email: telsch@inel.gov 